\title{
Patterns or mechanisms? Bergmann's and Rapoport's rule in moths along an elevational gradient
}

\author{
J. Beck ${ }^{1,2,8}$, H. C. Liedtke ${ }^{3}$, S. Widler ${ }^{2}$, F. Altermatt ${ }^{4,5}$, S. P. Loader6, R. Hagmann²,
} S. Lang 2 and K. Fiedler ${ }^{7}$

${ }^{1}$ University of Colorado, Museum of Natural History, 265 UCB, Boulder, CO 80309, USA

2 Institute of Biogeography, University of Basel, St. Johanns-Vorstadt 10, 4056 Basel, Switzerland

${ }^{3}$ Ecology, Evolution and Developmental Group, Department of Wetland Ecology, Estación Biológica de Doñana (CSIC), 41092 Sevilla, Spain

${ }^{4}$ Eawag: Swiss Federal Institute of Aquatic Science and Technology, Department of Aquatic Ecology, Überlandstrasse 133, 8600 Dübendorf, Switzerland

${ }^{5}$ Department of Evolutionary Biology and Environmental Studies, University of Zurich, Winterthurerstrasse 190, 8057 Zürich, Switzerland

${ }^{6}$ University of Roehampton, Holybourne Avenue, London, SW154JD, UK

7 University of Vienna, Division of Tropical Ecology \& Animal Biodiversity, Rennweg 14, 1030 Vienna, Austria

${ }_{8}$ Corresponding author.E-mail: jan.beck@colorado.edu

Keywords: Altitude, Body size, Elevation, Macrolepidoptera, Range size, Temperature variability.

\begin{abstract}
Bergmann's rule predicts increasing body sizes at higher elevations. The elevational Rapoport's rule predicts an increase of elevational range size with higher elevations. Both rules have often been related to effects of temperature. Larger bodies allow more efficient heat preservation at lower temperature, explaining Bergmann's rule. Higher temperature variability may select for adaptations that allow increased range sizes, explaining Rapoport's rule. The generality of both rules has been challenged and evidence towards explanatory mechanisms has been equivocal. We investigated temperature and its variability as explanations for Bergmann's and Rapoport's rule in moths along an elevation gradient in Switzerland. In particular, we tested for relationships between elevation, temperature and body size across almost 300 species of Macrolepidoptera along a gradient from 600 to $2400 \mathrm{~m}$ a.s.l. The gradient was resampled throughout the vegetation season, which allowed assessing temperature effects independently from elevation. We controlled analyses for covariate traits of moths and their phylogeny. We found a positive relationship between body size and elevation, but no link with temperature. Furthermore, there was no positive link between average elevation and elevational range, but there was between temperature variability and elevational range. We conclude that mechanisms other than temperature can lead to increasing body sizes with elevation (supporting Bergmann's pattern, but not the mechanism). Contrary to that, data support the mechanism for Rapoport's rule: high temperature variability is associated with large ranges. However, because temperature variability is not necessarily increasing with elevation, it may not always lead to the geographic pattern predicted.
\end{abstract}

Abbreviations: AIC-Akaike's Information Criterion; CO1-Cytochrome Oxidase subunit 1; MaxLRT-Maximum Local Temperature Range (experienced by a species); OLS-Ordinary Least Squares; PCoA-Principal Coordinates Analysis; pGLS-phylogenetic Generalized Least Squares; GLM-Generalized Linear Model; t_range-temperature range (experienced by a species).

\section{Introduction}

Variation in animal body size along environmental gradients has been investigated since Bergmann (1848), who proposed that larger body sizes occur at higher latitudes and in colder climates. Originally developed for endothermic mammals, Bergmann assumed that the heat-preserving geometry of larger bodies acts as mechanism behind the observed pattern. Many studies (e.g., Diniz-Filho et al. 2009, Meiri 2010) found corresponding patterns along latitudinal or elevational gradients, while specific predictions relating to the heat-preserving mechanism were only sometimes met (Rodríguez et al. 2008, Diniz-Filho et al. 2009). A number of studies have also investigated body size variation in ectotherms, some of them finding support for a negative temperature-body size pattern while others found no link or even a positive temperature-body size relationship (for interspecific studies, see e.g., Ashton and Feldman 2003, Brehm and Fiedler 2004, OlallaTárraga and Rodriguez 2007, Watt et al. 2010, Hu et al. 2011).

The thermoregulatory mechanism inherent to Bergmann's rule is less plausible to cause body size patterns in ectotherms (but see Zamora-Camacho et al. 2014). However, flying insect taxa represent a continuum from fully environment-controlled body temperatures through behavioural thermoregulation to active warming by muscle shivering (Heinrich 1993). Given that there is further variation in regard to thermoregulation between life stages (i.e., larvae and adults) within spe- 
cies (Kingsolver et al. 2011, Woods 2013) and since for most species physiological data are lacking, there is no synthetic theory available yet from which testable, insect-specific predictions on the interspecific relationship between environmental temperature and body size could be deduced. Gaston and Chown (2013) give an overview of the current knowledge on insect body sizes from a pattern-descriptive perspective.

Interspecific body size variation has been related to a number of other variables, for example starvation resistance in unpredictable habitat conditions (Blackburn and Hawkins 2004) or resource use (Brown and Maurer 1989). For moths with herbivorous larval stages, body size in multi-species comparisons was suggested to be linked to host plant type (herb vs. tree; Lindstroem et al. 1994, and references therein) and diet specialization (Davies et al. 2012). Additionally, body size in many organisms is phylogenetically conservative (Wiens et al. 2010, Gaston and Chown 2013) and as a consequence it may co-vary with other conserved traits. As such variables likely co-vary with climatic gradients, testing Bergmann's rule requires accounting for the interactions amongst a number of potentially confounding variables.

As in Bergmann's rule, temperature is also at the heart of an explanation of Rapoport's rule that predicts the geographic distribution of range sizes (note that we use the term 'rule' to pragmatically name a described pattern). Rapoport's rule describes a pattern where species from higher latitudes (i.e., cooler regions) have larger range sizes (Stevens 1989). A modification (or special case), which is treated in this study, is captured by the elevational Rapoport rule (Stevens 1992), which predicts larger elevational ranges (i.e., the range between lowest and highest occurrence) in species occurring at higher (i.e., cooler) elevations. Evidence for the generality of Rapoport's rule is weak in an elevational context (McCain and Knight 2013). Stevens (1989, 1992; based on ideas by Janzen 1967) proposed an increase of climatic variability with elevation as a potential mechanism for the Rapoport pattern. Species experiencing higher variability, he argued, would require adaptations that also allow them to occupy larger ranges. McCain (2009) confirmed Janzen's (1967) prediction by showing that vertebrates have smaller elevational ranges on non-seasonal tropical mountains than on seasonal mountains. She also reported larger ranges in the upper third of elevation gradients compared to the lower third. Further ideas on links between longer-term temperature variability and range size have been developed by several more authors (e.g., Dynesius and Jansson 2000, Sandel et al. 2011).

In the present study, we investigate the links between body size, abundance-weighted mean elevation of occurrence and elevational range size in moths along a gradient in the Swiss Alps. We identified, and attempt to address, three shortcomings of many earlier studies of this type (Appendix ES1, Table S1). (1) Many eco-morphological traits have the potential to affect the distribution of species, and ignoring them may lead to false conclusions regarding the investigated patterns. Therefore, we use a broad compilation of life history traits as covariates in analyses. (2) Body sizes, as well as emergent traits such as range size, may be linked to phy- logeny (Wiens et al. 2010). We control our analyses for confounding effects of phylogenetic non-independence using a molecular phylogeny of the taxa involved. (3) Many environmental variables are highly collinear with elevation, most prominently temperature. Elevational patterns are therefore often interpreted as temperature effects, but this assertion is notoriously difficult to test. Our data set consists of eight monthly replicate samples along the same elevation gradient. Because the studied moths are dormant through times of unsuitable climate and are only active during a limited fraction of the annual cycle, we can at least partly disentangle effects of habitat elevation per se from immediate temperature effects: Highly variant phenologies (across species) in a seasonal landscape lead to substantial decoupling of elevations of occurrence and the temperatures encountered.

Figure 1 illustrates the hypotheses tested in this study. If Bergmann's rule applies to our dataset, we expect that the mean elevation where species occur is positively related to its body size $(\mathrm{Hl})$. Furthermore, if Bergmann's rule is caused by the proposed thermoregulatory mechanism, we expect that elevation and temperature show the same statistical relationships (i.e., replacing elevation by temperature should recover similar patterns, but inversely so; H2). Rapoport's rule predicts that the elevational range size of species is positively related to the (average) elevation where species occur (H3). If the proposed mechanism for Rapoport's rule is valid, the elevational range size of species should be positively related to the temperature variability within which species were found ( $\mathrm{H} 4)$. Because the details of the proposed mechanism for the elevational Rapoport rule leave room for interpretation (Stevens 1992, McCain and Knight 2013), we tested two more specific variants of predictions: $(\mathrm{H} 4 \mathrm{a}$ ) a correlation of elevational range and the overall temperature range where a species was found (due to the adiabatic relationship this is tautological for species permanently exposed to local temperatures, but not for species that exhibit dormancy or migratory behaviour); and (H4b) a correlation of elevational range and the maximum temperature range that a species was exposed to at any local site.

\section{Methods}

\section{Moth occurrence, temperature and traits}

We sampled nocturnal Macrolepidoptera with automatic light traps along an elevation gradient from ca. 600 to $2400 \mathrm{~m}$ a.s.l. in the northern Swiss Alps, south of Lake Thun (Kiental, $\left.\mathrm{N} 46^{\circ} 35^{\prime}, \mathrm{E} 007^{\circ} 44^{\prime}\right)$. Traps attracted moths with UV light from dusk till dawn and lead them through a funnel into a bucket containing cotton fabric and an egg carton impregnated with a pyrethroid contact insecticide (Heath trap with light detector switch, Bioform article no. 1001-02, http://www.bioform.de; light output equivalent to $15 \mathrm{Watt})$. The immobilized moths were collected daily from the traps. Fourteen sites were simultaneously sampled over eight temporal replicates throughout the growing season (snow conditions permitting), i.e., from April until November 2008 (4-night sessions replicated every 4-5 weeks). Sites were chosen to represent 
near-natural vegetation types. In total, 396 trap-nights captured $~ 5900$ individuals, representing 9 families and 294 reliably identified species (plus some 'morphotypes' that were ignored in this study since no trait data could be attributed here). Abundance per species ranged from one to 533 specimens $($ mean $=18.3$, median $=4 ; 89$ species were represented by $\geq 10$ specimens; see robustness analyses below).

At each sampling location, temperature loggers took hourly measurements through the study period. In forested habitats loggers were placed in undergrowth trees at $1.5 \mathrm{~m}$ height, whereas above the tree line we positioned them under stones to avoid measurement artefacts through direct solar radiation. Details on field methodology, moth identification, and the elevational pattern of species diversity were reported in Beck et al. (2010).

For temperate-zone moths that spend a considerable time of the year in winter dormancy, it is mostly the temperature encountered during active life stages that affect their ecology and metabolism. In the absence of site- and species-specific life span data, we associated elevation and date of capture with the average local temperature of the four weeks preceding capture for every specimen. This temperature average should capture a significant part of the thermal conditions encountered by the late instars of the growing caterpillar, the pupal stage and the adult, while still producing relatively independent data (because captures were spaced at least four weeks apart). For each species we calculated elevation and temperature averages (weighted by their abundance at sites), elevational ranges (difference between highest and lowest elevation where a species was found) and temperature ranges (difference between highest and lowest temperature where a species was found, within the four-week interval prior to capture). Furthermore, we measured the maximum local temperature range that individuals of a species could have experienced at a single site within a four week-period (MaxLRT; based on hourly measures). As we generally worked with species-specific averages, weighted by individual numbers, rare vagrants in non-breeding habitats will not greatly affect these calculations. Our northern alpine study gradient is not waterlimited, so we did not expect relevant precipitation effects. Because temperatures measured during the 2008 field season might deviate from long-term climatic averages (which shaped ecological communities), we repeated analyses using 50-year climatic averages (from www.worldclim.org) to assure that this did not bias conclusions (see Electronic Supplement ES6).

Body sizes of specimens were measured with a calliper, taking standard metrics for Lepidoptera (i.e., forewing length, thorax width, and body length (measured from frons to end of abdomen)). Where available, we measured up to 10 specimens and used their average for analyses. Because some singleton specimens were damaged, we supplemented
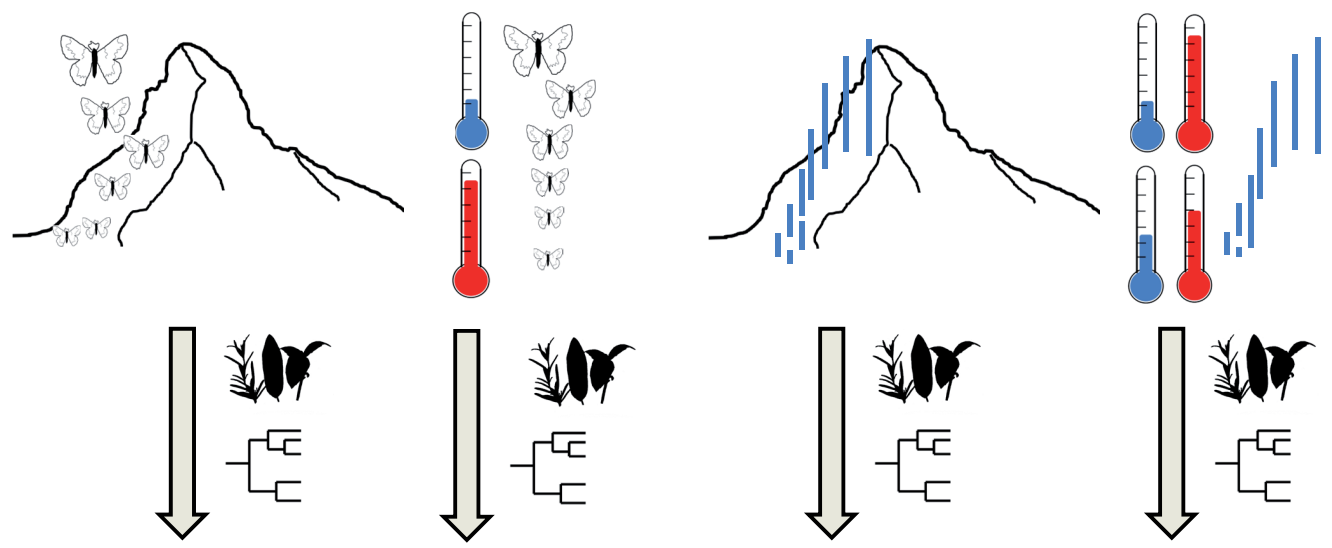

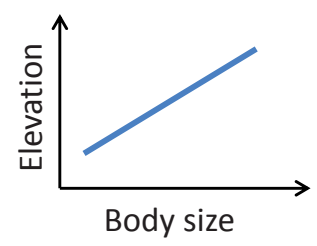

H1

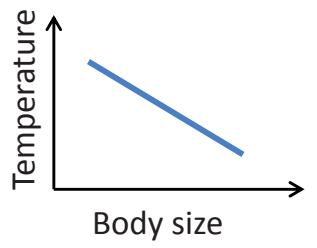

$\mathrm{H} 2$

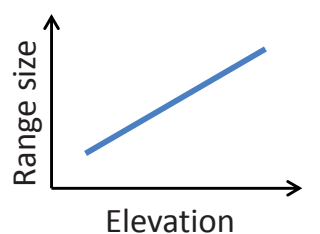

H3

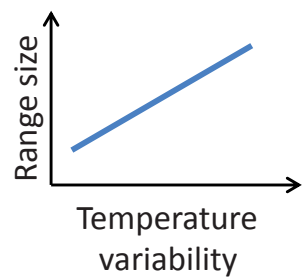

$\mathrm{H} 4$

Figure 1. Hypothetical relationships of traits and range properties of species. [H1] Larger species occur at higher elevations. After controlling for various covariate traits (symbolized by host plants) and phylogenetic effects, a positive relationship of body size with elevation emerges. [H2] Larger species occur in colder habitats while smaller species occur in warmer habitats. After controlling for traits and phylogeny, a negative body size-temperature relationship emerges. [H3] Species occurring at higher elevations have larger elevation ranges. After controlling for traits and phylogeny, a positive relationship of species' average elevation and their elevation range emerges. [H4] Species occurring at sites with higher temperature variability have larger elevation ranges. After controlling for traits and phylogeny, a positive relationship of the variability of temperatures encountered by a species and their elevation range emerges. 
our own measures with body sizes for 34 species from the literature (results did not change without these species included). The product of body length and thorax width, as an estimate of body volume and hence mass, was highly correlated with body length $\left(r^{2}=0.91\right)$, hence body length was used as the only size measurement in the analyses presented. Due to large family-specific differences in wing shape in Lepidoptera, we did not include forewing length into a proxy of body mass (correlation forewing-length vs. body length: $\left.r^{2}=0.66\right)$. Intraspecific trait variation is always a concern in comparative studies (Albert et al. 2010). However, with a large interspecific variation (body lengths ranging from 6.0 to $44.5 \mathrm{~mm}$ ) we judge effects of intraspecific variation to be relatively negligible (but see Sullivan and Miller 2007). We did not observe obvious within-species elevational body size trends in our collection; however, measuring and analysing body size variation on the individual level for many thousands of specimens was beyond the scope of this paper.

We used standard references for the central European moth fauna to extract species' ecological traits (Appendix: Electronic Supplement ES1). We extracted the following information for all species: Adult feeding (proboscis present/ absent), hibernation stage (egg/larva/pupa/adult; three migrant species were classified as "adult hibernating" for the purposes of this analysis), typical generation phenology (in three categories, see ES4 for details), the identity of larval host plants, and habitat preference (four categories: deciduous forest, coniferous and mixed forest, shrub, grassland and rocky sites). We analysed larval host plant associations only at high systematic level, i.e., plant orders (as suggested by preliminary analysis; classification followed APG 2009 and http://www.mobot.org/MOBOT/research/APweb/).

\section{Phylogeny}

Multi-gene molecular phylogenies of the higher taxonomic lineages of Lepidoptera have recently been published (Regier et al. 2013) but their taxon sampling was insufficient to cover all species included in our study. We followed a hybrid approach to provide the most reliable phylogenetic hypothesis for all species while accounting for missing data (both genes and taxa). Our phylogeny was assembled using a single mitochondrial gene for which published data were available (DNA barcoding region of CO1). The phylogeny was then constrained at tribe level based on more reliable conclusions of published multi-gene approaches that also included nuclear markers (Regier et al. 2013). Furthermore, we inserted taxa for which no $\mathrm{CO} 1$ sequence data were available into the tree based on systematic classifications. Details on methodology and the resulting working phylogeny for our taxa are presented in ES2 and ES3.

\section{Statistical analyses}

We analysed data in a comparative framework, i.e., using each species as one data point and traits as well as range properties as variables (Sanders 2002). We prefer this spe- cies-focus to the more commonly applied location-focused analyses, because (a) it allows a straightforward control for phylogeny and covariant traits, (b) it avoids random effects at sites with very few species (e.g., high elevations or samples taken very early or late in the year) exerting a strong impact on recovered patterns, and (c) it reduces pseudo-replication from species occurring at multiple sites.

Body length data were $\log _{10}$-transformed prior to analysis. We applied principal coordinates ordination (PCoA) to host plant orders, retaining $>48 \%$ of variance with four axes that were used for further analyses. Multinomial categorical data were coded as binomial dummy variables; we assured that dummy variables were not strongly correlated to other dummies of the same trait category (all $r^{2}<0.25$ ).

We used species' average elevation and their elevational range, and the temperature and its range experienced by species, as response variables in our models. In other words, our analyses tested how well traits could predict where a species occurs (rather than how environmental conditions predict traits). Note that all correlation analyses are unspecific to what actually is cause and what is effect.

We investigated relationships of responses with predictor variable groups (body size, voltinism, hibernation stages, proboscis, number of host plant orders, spectrum of host plant orders (PCoA axes), habitat preference) in various linear combinations in Generalized Linear Models (GLMs) and carried out model selection based on Akaike's information criterion (AIC; Burnham and Anderson 2002). Because candidate models were often equivalent $(\triangle \mathrm{AIC}<2)$, we used model averaging to judge the strength of coefficients.

We only used traits (apart from body size) as co-variates for rigorously testing our hypotheses on body size, elevation and temperature. To account for this, we neither report, nor base any conclusions on, model p-values for trait effects apart from the hypotheses we were testing, hereby avoiding statistical issues related to pre-screening variable contributions (Forstmeier and Schielzeth 2011).

\section{Testing for Bergmann-patterns}

We first tested body length against average elevation (as a response variable, see above) by univariate OLS regression. We also tried fitting a quadratic polynomial function of body length to assure we were not missing unimodal effects. Then we included other trait groups in GLMs and carried out model selection to investigate whether these traits explain part of the body length vs. elevation relationship or any additional variance.

We based conclusions for $\mathrm{H} 1$ on whether or not body size was included in the best model (lowest AIC) and on AICweighted parameter averaging of the body size-effect in the model. The analyses were repeated using temperature instead of elevation as a response variable. This tests relationships with ambient temperature ( $\mathrm{H} 2$ ) relatively independently from elevation (linear regression of species' average elevation vs. average ambient temperature: $r^{2}=0.09$ ). 


\section{Testing for elevational range patterns}

To test for Rapoport's rule (H3), we followed a procedure similar to the one described above, but used elevational range as response variable and average elevation as a predictor. However, theory predicts a geometric artefact leading to a unimodal pattern (the 'mid-domain effect', Colwell and Hurtt 1994; see also robustness analyses below and ES5). To control for this we carried out analyses on the Rapoport pattern using only the smaller half of range sizes, which removed such effects (cf. McCain and Knight 2013). Average elevation was then replaced by temperature range measures as a predictor of elevational range (testing $H 4$, in both alternative interpretations (see Introduction)). For consistency, we carried out temperature range-analyses with the full dataset as well as with only the smaller half of elevational range sizes.

\section{Controlling for phylogenetic effects}

Pagel's $\lambda$ (Pagel 1997) was used to measure phylogenetic signal for body size, temperature and elevation data. We repeated univariate and 'best' models if $\lambda \geq 0.2$, using a phylogenetic generalized least square model (pGLS; Paradis et al. 2004). Preliminary explorations best supported models with an Ornstein-Uhlenbeck model of trait evolution (vs. Brownian motion), so we present these results. Because in our phylogenetic tree branch length variances are not homogenous (ES3), we included a fixed offset weight to pGLS models.

\section{Robustness}

We repeated analyses with various subsets of data to evaluate the robustness of conclusions. In particular, we excluded 'rare' species with measured elevational ranges of zero (i.e., species found only at one site, hence all singletons and most doubletons). This helps reducing undersampling effects on elevational position and range size data. As an alternative approach, we also excluded all species that were represented by $<10$ individuals. We also excluded species that fly predominantly in spring (average occurrence before June 1st), as larval development in these may have occurred (partly) in the previous year, invalidating our 'four weeks previous to collection' approach.

All statistical analyses were carried out in R v.2.15.2 (http:// www.r-project.org/; packages AICcmodavg (https://cran.rproject.org/web/packages/AICcmodavg/AICcmodavg.pdf), APE (Paradis et al. 2004), nlme (http://CRAN.R-project.org/ package $=$ nlme), geiger (Luke et al. 2008), phytools (https:// cran.r-project.org/web/packages/phytools/phytools.pdf).

\section{Results}

Body size and elevation: Bergmann pattern (H1)

Average elevation increases with species' body size (Fig. $2)$, but the univariate effect is weak $\left(N=294, r^{2}=0.052\right.$, $\mathrm{p}<$

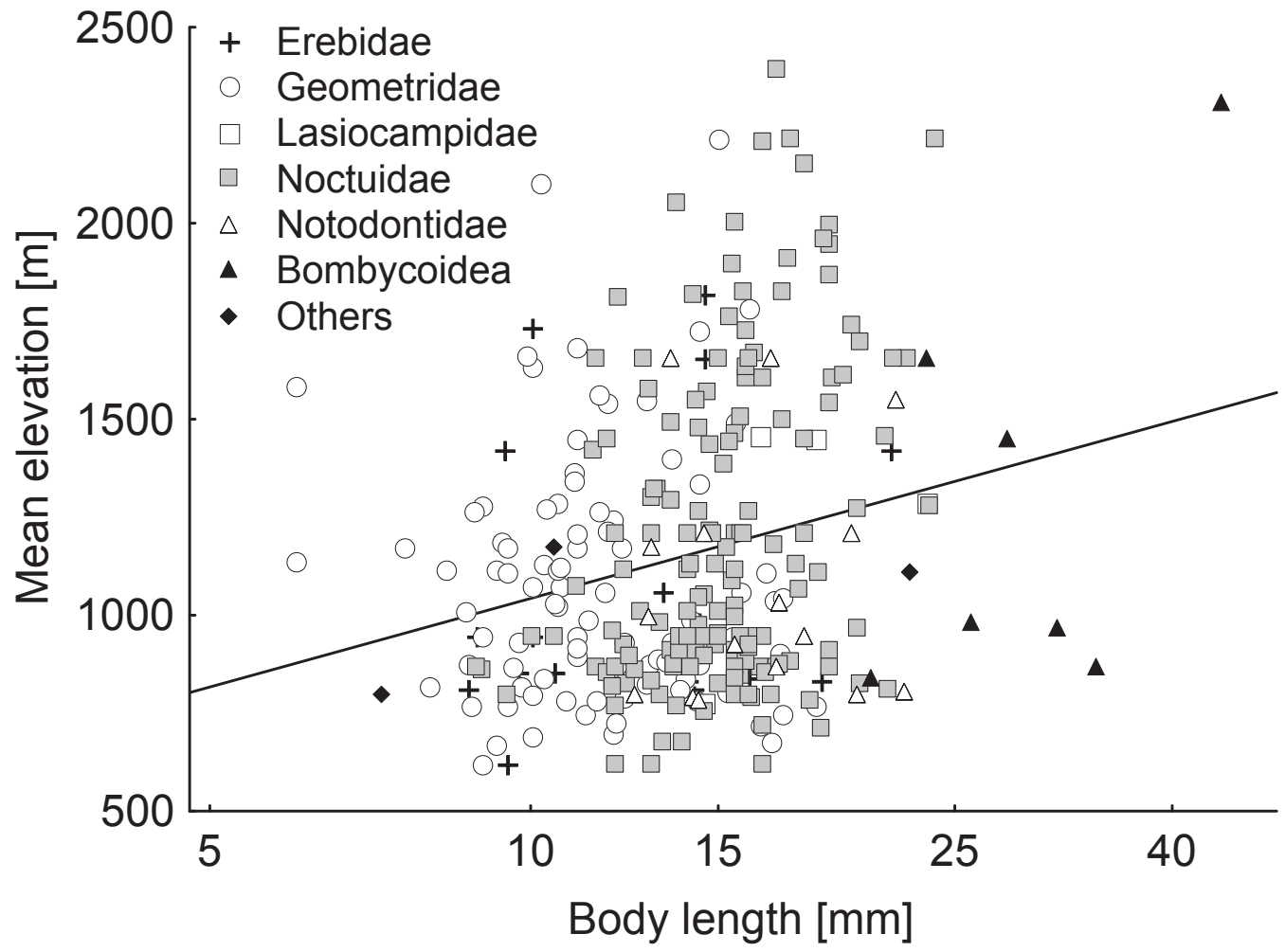

Figure 2. Body size of moth species (log-scaled x-axis) is positively correlated to the average elevation of their (abundance-weighted) occurrence, but the relationship is weak $\left(r^{2}=0.05\right)$. Higher systematic groupings are indicated. Data for all 294 species are shown. Separate analyses for the two most species-rich families revealed significant univariate $\left(r^{2}=0.11\right)$ and multivariate body size effects for Noctuidae ( $N=155$ species, mirroring overall patterns), but no effects for Geometridae ( $N=89$ species). 
Table 1. (A) Model selection for average elevation of occurrence, ranked by AIC ( $\mathrm{N}=294$ species for all models). Only the top five models are shown, see ES4 (Table S1) for full data. (B) Details for the 'best model' (highest AIC weight). Note that we only present assessments of statistical significance for body size effects (see Methods). Data for a pGLS-version of the 'best model' are also given.

\begin{tabular}{|c|c|c|c|c|}
\hline (A) Candidate model & AIC & $\triangle \mathrm{AIC}$ & AIC weight & pGLS AIC \\
\hline size + hosts + habitat + hibernation & 4263.1 & 0.0 & 0.80 & 3912.0 \\
\hline size + hosts + hibernation & 4266.7 & 3.6 & 0.13 & \\
\hline full model & 4268.5 & 5.4 & 0.05 & \\
\hline size + hosts & 4271.1 & 7.9 & 0.02 & \\
\hline size + hosts + hostbreadth + proboscis & 4275.0 & 11.9 & 0.00 & \\
\hline (B) Best model $\left(\mathrm{R}^{2}{ }_{\text {adj }}=0.222\right)$ & Coefficient $\pm \mathrm{SE}$ & $\mathrm{t}$ & pGLS coefficient \pm SE & pGLS t \\
\hline (Intercept) & $533.2 \pm 218.8$ & 2.44 & $492.2 \pm 227.6$ & 2.162 \\
\hline size $\left[\mathrm{mm}, \log _{10}\right.$-transformed] & $* * * 681.3 \pm 175.3$ & 3.89 & $* * * 718.7 \pm 184.2$ & 3.902 \\
\hline hosts_factor1 & $-80.1 \pm 25.9$ & -3.09 & $-90.4 \pm 26.3$ & -3.433 \\
\hline hosts_factor2 & $99.2 \pm 38.2$ & 2.59 & $108.8 \pm 37.4$ & 2.908 \\
\hline hosts_factor3 & $63.9 \pm 48.8$ & 1.31 & $70.8 \pm 47.8$ & 1.482 \\
\hline hosts_factor4 & $-89.4 \pm 53.7$ & -1.66 & $-83.8 \pm 53.5$ & -1.565 \\
\hline hibernation_adult & $0^{+}$ & & $0^{+}$ & \\
\hline hibernation_egg & $-248.3 \pm 87.0$ & -2.86 & $-197.7 \pm 87.5$ & -2.260 \\
\hline hibernation_larva & $-96.7 \pm 75.8$ & -1.28 & $-90.1 \pm 78.4$ & -1.149 \\
\hline hibernation_pupa & $-158.1 \pm 76.0$ & -2.08 & $-146.4 \pm 79.4$ & -1.845 \\
\hline habitat_deciduous & $0^{+}$ & & $0^{+}$ & \\
\hline habitat_coniferous\&mixed & $-87.3 \pm 45.1$ & -1.94 & $-105.0 \pm 43.7$ & -2.402 \\
\hline habitat_shrub & $-22.4 \pm 43.4$ & -0.52 & $-16.1 \pm 42.0$ & -0.384 \\
\hline habitat_grassland\&rocky & $73.8 \pm 50.7$ & 1.46 & $62.7 \pm 49.4$ & 1.269 \\
\hline
\end{tabular}

*) $\mathrm{p}<0.05 ; * *) \mathrm{p}<0.01: * * *) \mathrm{p}<0.001 ;{ }^{+}$) zero by default

Modelled effects and their abbreviations: 'size' $=\log _{10}$ (body length, in $\mathrm{mm}$ ); 'hosts' = larval host plant orders, expressed by 4 factors from PCoA (see Methods), 'hostbreadth' = number of larval host plant orders utilized; 'habitat' = literature-derived habitat preference (4 categories, coded as 3 dummy variables), 'hibernation' = one of four life stages in which hibernation occurs (coded as 3 dummy variables); 'voltinism' = one of three voltinism strategies (coded as 2 dummy variables); 'proboscis' = presence of a proboscis (i.e., adult feeding). The full model consists of the variables size, hosts (4 factors), hostbreadth, habitat ( 3 dummies), hibernations ( 3 dummies), voltinism ( 2 dummies), and proboscis (i.e., coded by 15 variables).

0.001; there was no indication for a unimodal effect). A positive effect of body size is part of the 'best' multivariate model, and its coefficient remains almost unchanged when considering the 'best model' (Table 1) or the averaged coefficient $\pm \mathrm{SE}$ $=680.3 \pm 175.9$ (the $95 \%$ confidence interval of the averaged coefficient does not overlap zero).

Body size carries extremely strong phylogenetic signal $(\lambda=1.00)$ whereas this is weaker for average ambient temperature $(\lambda=0.634)$ and for elevation $(\lambda=0.298)$. pGLS confirmed the significance of the univariate relationship of (log-transformed) body size and elevation (slope $\pm \mathrm{SE}=$ $638.7 \pm 197.9, \mathrm{t}=3.23, \mathrm{p}=0.001)$ as well as the body size effect in the 'best' multivariate model (Table 1).

\section{Temperature as mechanism for the Bergmann pattern (H2)}

Replacing average elevation with average temperature as a response variable did not recover a univariate relationship
$\left(N=294, r^{2}<0.001, \mathrm{p}=0.798\right)$; there was no support for a unimodal model either $\left(r^{2}=0.006\right)$. AIC-evaluation of multivariate models, predicting average temperature from species' traits, renders the full model except body size as 'best model' (ES4). The best model explains average temperature approximately as good $\left(\mathrm{R}^{2}{ }_{\text {adj }}=0.216\right)$ as the best model for average elevation does $\left(\mathrm{R}^{2}{ }_{\text {adj }}=0.222\right)$. The full model $(\triangle \mathrm{AIC}=1.02)$ explains no more variability. The averaged coefficient $\pm \mathrm{SE}$ for body size on temperature is non-significantly positive $(0.97 \pm 1.44)$; note that a negative coefficient would be expected according to the hypothesized mechanism for Bergmann's rule. Remodelling the univariate, 'best' and full multivariate models with pGLS did not change conclusions (i.e., no significant relationships were found; not shown in detail).

Robustness analyses mostly supported conclusions on Bergmann's rule and its mechanism (ES5), as did analyses based on long-term averages instead of own temperature measurement (ES6). 


\section{Elevation and elevational range: Rapoport pattern (H3)}

Relationships between average elevation and elevational range size were investigated for the $N=146$ species representing the smaller half of range sizes. Contrary to the hypothesis $(H 3)$, we found a significantly negative, though weak link between elevation and elevational range in a univariate correlation $\left(r^{2}=0.080, \mathrm{p}=0.002\right)$. A quadratic model was slightly better $\left(r^{2}=0.109\right)$, but it still modelled a monotonically negative relationship within the data range (not shown). Elevation is contained in the 'best model' as well as in three out of five 'good models' ( $\triangle \mathrm{AIC}<2$; ES4), but parameter averaging again indicates a negative link (averaged coefficient $\pm \mathrm{SE}=-0.04 \pm 0.02 ; 95 \%$ confidence intervals (CI) for the averaged coefficient overlap zero). The best model contains average elevation and host plant orders (four factors; $\mathrm{R}^{2}{ }_{\text {adj }}=0.197$ ), but the (negative) effect of elevation is not significant in the model $(\mathrm{t}=-1.67, \mathrm{p}=0.097)$.

There was only weak phylogenetic signal in elevational ranges ( $\lambda=0.295$ for smaller-ranged half of species) and elevational averages $(\lambda=0.278)$, and univariate pGLS confirmed OLS results of a negative relationship between average elevation and elevational range $(\mathrm{t}=-3.54, \mathrm{p}<0.001)$.

However, when including all species in the analysis (i.e., not excluding the larger-ranged half), we did find a weak, but positive relationship between average elevation of species and their elevational range size (presented in ES5). We note that this is at least partially due to the geometric artefact of the mid-domain effect (see Methods and ES5).

\section{Temperature variability as mechanism for the Rapoport pattern (H4)}

We found a strong positive correlation between species' range of occurrence temperatures and their elevational range (hypothesis $H 4 a$ : Fig. $3 ; N=294, r^{2}=0.582, \mathrm{p}<0.001$ ).
Model selection has occurrence temperature range as part of all 'good' models (Table 2) with the averaged coefficient \pm SE $=120.6 \pm 5.6$ ( $95 \% \mathrm{CI}$ does not overlap zero). Repeating these analyses with only the smaller half of range sizes (for consistency with analyses above) confirmed our conclusion of a temperature range effect (univariate: $N=146$ species, $r^{2}=0.377$, $\mathrm{p}<0.0001$; averaged coefficient \pm SE from model selection $=$ $32.6 \pm 4.0,95 \%$ CI does not overlap zero; the best model contains temperature range $(\mathrm{p}<0.0001)$ as well as host plants, habitat and hibernation strategy, $\mathrm{R}_{\text {adj }}=0.472$ ).

When using maximum monthly local temperature ranges (MaxLTR), we also found a significant positive correlation with elevational range (hypothesis H4b: Fig. $3 ; N=294, r^{2}=$ $0.316, \mathrm{p}<0.001$ ). Model selection (Table 2) had MaxLTR contained in all good models with the averaged coefficient $\pm \mathrm{SE}$ $=52.36 \pm 4.5$ (95\%CI does not overlap zero). Repeating these analyses with only the smaller half of range sizes (for consistency with analyses above), however, did not recover a significant univariate relationship of elevational range and MaxLTR $\left(N=146, r^{2}=0.011, \mathrm{p}=0.226\right)$ although model selection recovered a significant, positive effect in the best model $\left(\mathrm{R}^{2}{ }_{\mathrm{adj}}\right.$ $=0.211$, containing MaxLTR and hostplant factors) as well as from the averaged coefficient $(5.2 \pm 2.3 ; 95 \% \mathrm{CI}$ does not overlap zero). Analyses based on long-term climate averages also supported these results (ES6).

Because of only weak phylogenetic signal in data (elevational ranges: $\lambda=0.140$; $t$ range: $\lambda=0.044$; MaxLTR: $\lambda=0.161$; all for full data) models were not retested with pGLS. Robustness analyses mostly supported results (but not the MaxLRT effect under rigorous data exclusion conditions; ES5). Full data as used in these analyses are published as ES8.

\section{Discussion}

Key findings of our analysis are a positive, but weak relationship between body size and elevation (supporting $H 1$,
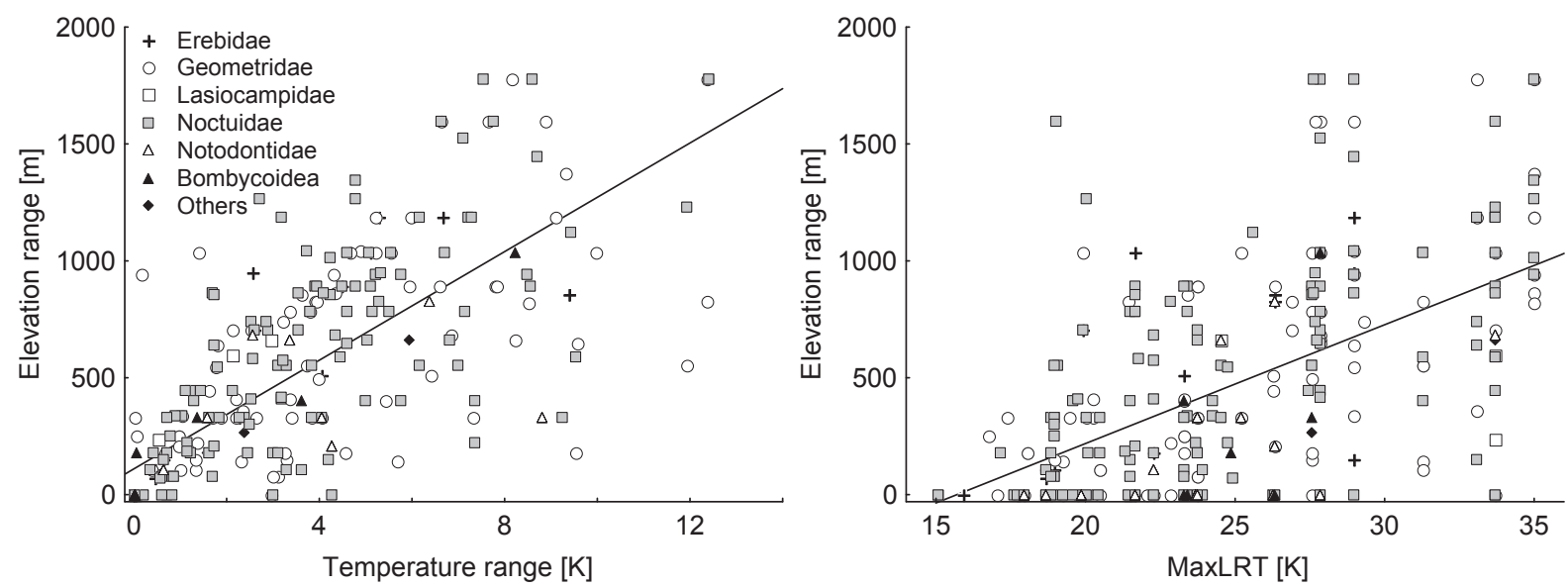

Figure 3. The elevational range of species is correlated to (left) the range of temperatures where the species were found $\left(r^{2}=0.58\right)$ as well as (right) the maximum monthly local temperature range (MaxLRT, $r^{2}=0.32$ ). Higher systematic groupings are indicated. Data for all 294 species are shown. 
Table 2. (A) Model selection and 'best model' explaining elevational range of species from temperature range ( $t$ range $)$ and other traits (see Table 1 for abbreviations; only top five models are shown, see Appendix for full data). Analyses for all species are shown. (B) Equivalent results for effects of maximum local temperature range (MaxLTR) experienced by a species.

\begin{tabular}{|c|c|c|c|}
\hline (A) Candidate model & AIC & $\Delta \mathrm{AIC}$ & AIC weight \\
\hline t_range + hibernation & 4155.4 & 0.0 & 0.78 \\
\hline t_range + hibernation + voltinism & 4159.1 & 3.7 & 0.12 \\
\hline t_range + hosts + hibernation & 4160.7 & 5.2 & 0.06 \\
\hline t_range + hosts + habitat + hibernation & 4161.3 & 5.9 & 0.04 \\
\hline full model & 4170.4 & 14.9 & 0.00 \\
\hline Best model $\left(\mathrm{R}_{\text {adj }}^{2}=0.627\right)$ & Coefficient \pm SE & $\mathrm{t}$ & \\
\hline (Intercept) & $-13.1 \pm 63.8$ & -0.21 & \\
\hline t_range & $* * * 120.7 \pm 5.5$ & 21.86 & \\
\hline hibernation_adult & $0^{+}$ & & \\
\hline hibernation_egg & $107.1 \pm 73.7$ & 1.45 & \\
\hline hibernation_larva & $215.2 \pm 62.5$ & 3.44 & \\
\hline hibernation_pupal & $0.9 \pm 63.4$ & 0.01 & \\
\hline (B) Candidate model & AIC & $\Delta \mathrm{AIC}$ & AIC weight \\
\hline MaxLTR + hosts + habitat + hibernation & 4313.7 & 0.0 & 0.85 \\
\hline full model & 4318.9 & 5.2 & 0.06 \\
\hline MaxLTR + hosts + hibernation & 4319.9 & 6.2 & 0.04 \\
\hline MaxLTR + habitat & 4320.4 & 6.7 & 0.03 \\
\hline MaxLTR + hostbreadth & 4321.8 & 8.1 & 0.02 \\
\hline Best model $\left(\mathrm{R}_{\text {adj }}^{2}=0.375\right)$ & Coefficient \pm SE & $\mathrm{t}$ & \\
\hline (Intercept) & $-958.7 \pm 140.8$ & -6.81 & \\
\hline MaxLTR & $* * * 52.3 \pm 4.5$ & 11.74 & \\
\hline hosts_factor1 & $58.6 \pm 27.7$ & 2.11 & \\
\hline hosts_factor2 & $-22.9 \pm 41.5$ & -0.55 & \\
\hline hosts_factor3 & $17.7 \pm 53.2$ & 0.33 & \\
\hline hosts_factor4 & $38.1 \pm 58.9$ & 0.65 & \\
\hline hibernation_adult & $0^{+}$ & & \\
\hline hibernation_egg & $73.7 \pm 96.2$ & 0.77 & \\
\hline hibernation_larva & $41.3 \pm 82.4$ & 0.50 & \\
\hline hibernation_pupa & $-134.9 \pm 82.5$ & -1.63 & \\
\hline habitat_deciduous & $0^{+}$ & & \\
\hline habitat_coniferous\&mixed & $77.8 \pm 49.7$ & 1.57 & \\
\hline habitat_shrub & $111.6 \pm 47.4$ & 2.36 & \\
\hline habitat_grassland\&rocky & $98.2 \pm 55.3$ & 1.78 & \\
\hline
\end{tabular}

*) $\mathrm{p}<0.05 ; * *) \mathrm{p}<0.01: * * *) \mathrm{p}<0.001 ;{ }^{+}$) zero by default

The full model consists of the variables t_range (A) or MaxLTR (B), size, hosts (4 factors), hostbreadth, habitat (3 dummies), hibernation (3 dummies), voltinism ( 2 dummies), and proboscis (i.e., coded by 16 variables).

as expected by Bergmann's rule), but no evidence for a direct link between body size and ambient occurrence temperature that would support a thermoregulatory explanation (i.e., not supporting H2). Furthermore, there is no evidence for a positive link between average elevation and elevational range of moth species (i.e., no support for $H 3$, no Rapoport pattern), but a positive relationship between occurrence temperature range and elevational range was detected (i.e., supporting $H 4$; Janzen 1967), irrespectively of what calculation of temperature range was used. Results were robust to the exclusion of rare species and of spring-flying species. Furthermore, conclusions are not confounded by covariant traits (of those included in analyses) or phylogenetic signal. 


\section{Bergmann's rule: body size, elevation and temperature}

We found a change of body sizes with elevation that was clearly not related to the ambient temperature that the moths had experienced during the month before capture. Independently from us, G. Brehm (pers. comm.) reached the same conclusions for moths from a Neotropical elevation gradient. Although a mechanism related to heat conservation was not necessarily expected for the (mostly) ectotherm organisms studied here, this result is of particular significance methodologically. Many gradient studies cannot, by design, find an elevational pattern that is not also correlated to temperature, due to the adiabatic link of temperature with elevation. But organisms that are not active throughout the year (i.e., avoid thermally adverse parts of the year by dormancy or migration) may experience physiologically relevant temperatures vastly different to the annual average at a given elevation. Despite the plausibility of the thermoregulatory explanation in endotherms, empirical data of elevational variation in body size may sometimes have been over-interpreted as evidence for a thermal mechanism.

Our findings raise the question of what other environmental factors could affect interspecific body size variation along elevational gradients. Some hypotheses to be tested in future studies are (a) an elevational change in mortality (Brehm and Fiedler 2004, but see Brehm et al. 2013), where lower mortality allows longer (larval) life spans, hence longer growth phases that allow attaining larger bodies; (b) the frequency of 'catastrophic' weather extremes may be more common at higher elevations; large species may be physiologically more robust, e.g., because they have more resources to survive in shelter without feeding (Blackburn and Hawkins 2004); or (c) large species may be more mobile, which allows rapid re-colonization after local extinction due to catastrophic events; high-elevation habitats may also be more fragmented, thus requiring higher mobility to maintain dispersal between populations; (d) if total individual number (across all species) declines with elevation faster than productivity (i.e., biomass) does, larger average body sizes could result (Longino and Colwell 2011); (e) larger wingspan has been interpreted as an adaptation to low air pressure at high elevation (in birds; Lee et al. 2008). Some of these hypotheses were also supported in intraspecific studies.

Bergmann (1848) proposed his negative temperature vs. body size relationship specifically for endotherms, but hypothetical mechanisms for both negative and positive links have been conceived for ectotherms as well. For example, opportunistic thermoregulation through flight muscle shivering occurs as a strategy in several flying insect taxa (Casey and Joos 1983), which may favour larger species in cold environments (Zamora-Camacho et al. 2014). Intra-specifically, development in cooler temperature has been found to lead to larger individuals in some species (Fischer and Fiedler 2002, Zuo et al. 2012). Chown et al. (2002), however, argued for the opposite effect with warmer temperatures leading to faster growth and hence larger size. Such uncertainty in theoretical expectations suggests that not finding any temperature-body size correlation across multiple unrelated species in this study is not particularly surprising.

A rather unexpected finding, however, was the stability of results to phylogeny-controlled models, given that body size in moths was highly conserved phylogenetically. A common perception of moth collectors is a shift in dominance with increasing elevation among the most abundant (and speciose) families, with geometrids being common in the lowlands and noctuids more common higher up on the mountain. We are not aware of quantitative data from the Alps that supports this; our own data suggest only a non-significant trend in this direction (not shown). As noctuids are typically larger, we had expected a priori a substantial phylogenetic effect on body size variation across the elevational gradient.

\section{The elevational Rapoport rule: elevation, elevational range, and temperature variability}

Rapoport's rule, latitudinal or elevational, has received mixed support in previous empirical studies (McCain and Knight 2013, Tomašových et al. 2015). Furthermore, an exact mechanism based on short-term temperature variability (Janzen 1967, Stevens 1992) has yet to be clearly formulated. Our approach of comparing species instead of spatial units allowed the consideration of two different interpretations of such a link: The full temperature spectrum experienced by a species, or alternatively the largest range experienced at any local site (by a given species). The former leads to an intuitive link with range size (species with a wider thermal niche should, on average, be found at more sites with differing conditions). The latter tests the hypothesis in a stricter evolutionary sense, as it implies the assumption that each species is adapting to the maximum local range and then spreads from there to other sites with permitting temperatures.

We found evidence for correlations of elevational and temperature range. However, we did not find evidence for a positive correlation between average elevation and elevational range, i.e., the elevational Rapoport pattern. There are several potential reasons for this. One likely cause is that the distribution of temperature ranges in the landscape may be different to what is commonly assumed. While it is beyond the scope of this paper to investigate the general geographic pattern of temperature variability, we found that the commonly held assumption of higher variability with increasing elevation does not seem well supported in the meteorological literature (Linacre 1982, Jackson and Forster 2010). Analyses of our own temperature measurements even revealed a negative link between elevation and temperature range, and preliminary global mapping based on interpolated climate data indicated an inconsistency of patterns from different regions, and negative relationships for many mountain ranges (including the Alps; ES7).

Declining temperature range with elevation, together with our finding of a negative correlation between average elevation and elevational range (see Results), suggests that a mechanism affecting elevational range size through temperature variability (sensu Stevens 1992, Janzen 1967) might 
be valid. However, because temperature variability does not follow a consistent pattern with elevation, this does not necessarily lead to the elevational Rapoport pattern. In line with this conclusion, it has been suggested that the high variability in the prevalence and direction of elevational range size patterns should lead future research towards direct analyses of how traits and environmental conditions shape range size distributions (McCain and Knight 2013).

However, there are also potential methodological causes for divergent results. While some studies have used, as we did, a comparative, species-based approach to study Rapoport's rule (e.g., Sanders 2002, Ruggiero and Hawkins 2006), most have analysed aggregated range sizes per elevation. When correlating median range sizes of all species recorded per sampling site to the elevation of sites (following the method of Stevens 1992), we found a positive elevation-range size pattern $\left(r^{2}=0.81\right)$. Adopting McCain and Knight's (2013) method of correlating the frequency of small-ranged species (lower quartile, to avoid the mid-domain effect) to the elevation of sites also led to results as predicted by Rapoport's rule (i.e., a decline of the frequency of small-ranged species with elevation, $r^{2}=0.32$ ). We see several potential reasons for this discrepancy. Undersampling of local communities, overrepresentation of common taxa and spatial autocorrelation could affect site-based analyses (favouring species-based analyses). However, deriving species-level range properties for species from one local gradient could also introduce error. Using regional data from literature could in part account for this (although this is rarely possible for environmental variables such as temperature), but this raises the question of local applicability. It is beyond the scope of this paper to resolve whether, and with what strength, these (and other) effects explain inconsistencies between site-level and species-level approaches. At this point, we only note that comparing results from studies applying different methods is not straightforward - they might each have their own biases and could subtly address different questions.

\section{Light trapping along a single gradient}

Several caveats must be highlighted for the interpretation of our results. Data were sampled along a single elevational gradient, with all its possible idiosyncrasies of topography, local climate and habitat distributions. Among these idiosyncrasies, our gradient was located in the temperate zone of the world, where all elevational ecological patterns are possibly more blurred than along less seasonal tropical gradients (Janzen 1967). Although earlier studies also measured elevational ranges along single gradients (McCain 2009, McCain and Knight 2013) the data for particular species may be different when measured on another gradient. In the absence of spatial replicates we cannot assess how much variation in emergent patterns and correlations this would cause. Despite detailed faunistic records for the central European moth fauna, there is paucity in comparable, systematic field studies along elevational gradients. Assessments of species' elevation ranges may also be erroneous due to undersampling (i.e., not finding rare species at a site although they do occur there) but robustness analyses (excluding very rare taxa) supported our general conclusions.

Furthermore, light trapping is biased with regards to activity times, sex, and potentially even taxonomic identity (Truxa and Fiedler 2012, Merckx and Slade 2014), and the activity of moths is affected by weather conditions during trap nights (but note that our temperature analyses refer to longterm averaging, not to single trap nights). Results therefore do not necessarily refer to all species and specimens in the landscape, but to the subset that was attracted and captured by this method. However, this caveat is shared with all other methods employed in ecological field studies that depend on animal activity rather than measuring 'true' abundances.

We found only relatively weak univariate correlations between some investigated properties of species, and even multivariate models left a lot of variance unaccounted for. This may appear as a failure of the investigated hypotheses (McCain and Knight 2013), as the uncovered links do not allow for reliable predictions. However, weak but consistent relationships are still relevant to identify evolutionary or ecological mechanisms even if obscured by local or taxonspecific idiosyncrasies and sampling error. It may be overly optimistic to expect a single mechanism explaining the distribution of highly aggregated traits such as range properties and average body size.

\section{Conclusions}

Mechanisms other than heat-preserving adaptations can lead to Bergmann patterns of body size with elevation in ectotherms, and potentially also in endotherms. The default of equating elevational (or latitudinal) variation with a causality of temperature may not always be warranted, especially if organisms can 'escape' periods of unsuitable temperatures by dormancy or migration. Conversely, thermal niche breadth was positively related to range size, which supports a suspected mechanism shaping range size distributions - but the consequent geographic placing of range sizes did not follow the pattern predicted by the elevational Rapoport rule, possibly because increasing temperature variability at higher elevations occurs less consistently in mountains than often assumed in ecology.

Acknowledgements: Thanks to C. McCain for helpful discussions and advice on literature and presentation. Field work was supported financially by a grant from Freiwillige Akademische Gesellschaft Basel (to JB). HCL is funded by the Swiss National Science Foundation (grant P2BSP3_158846).

\section{References}

Albert, C.H., W. Thuiller, N.G. Yoccoz, A. Soudant, F. Boucher, P. Saccone and S. Lavorel. 2010. Intraspecific functional variability: extent, structure and sources of variation. J. Ecol. 98 : 604-613.

APG-Angiosperm Phylogeny Group. 2009. An update of the Angiosperm Phylogeny Group classification for the orders and 
families of flowering plants: APG III. Bot. J. Linn. Soc. 161: 105-121.

Ashton, K.G. and C.R. Feldman. 2003. Bergmann's rule in nonavian reptiles: turtles follow it, lizards and snakes reverse it. Evolution 57: 1151-1163.

Beck, J., F. Altermatt, R. Hagmann and S. Lang. 2010. Seasonality in the altitude-diversity pattern of Alpine moths. Basic Appl. Ecol. 11: 714-722.

Bergmann, C. 1848. Über die Verhältnisse der Wärmeökonomie der Thiere zu ihrer Grösse. Göttinger Studien 1: 595-708.

Blackburn, T.M. and B.A. Hawkins. 2004. Bergmann's rule and the mammal fauna of northern North America. Ecography 27: $715-724$.

Brehm, G. and K. Fiedler. 2004. Bergmann's rule does not apply to geometrid moths along an elevational gradient in an Andean montane rain forest. Glob. Ecol. Biogeogr. 13: 7-14.

Brehm, G., P. Strutzenberger and K. Fiedler. 2013. Phylogenetic diversity of geometrid moths decreases with elevation in the tropical Andes. Ecography 36: 1247-1253.

Brown, J.H. and B.A. Maurer. 1989. Macroecology: The division of food and space among species on continents. Science 243: $1145-1150$.

Burnham K.P. and D.R. Anderson. 2002. Model Selection and Multimodel Inference: A Practical Information-theoretic Approach. Springer, Berlin.

Casey, T.M. and B.A. Joos. 1983. Morphometrics, conductance, thoracic temperature, and flight energetics of noctuid and geometrid moths. Physiol. Zool. 56: 160-173.

Chown, S.L., A. Addo-Bediako and K.J. Gaston. 2002. Physiological variation in insects: large-scale patterns and their implications. Comp. Biochem. Physiol. (B) 131: 587-602.

Colwell, R.K. and G.C. Hurtt. 1994. Nonbiological gradients in species richness and a spurious Rapoport effect. Am. Nat. 144: 570-595.

Davies, R.B., E. Ounap, J. Javois, P. Gerhold and T. Tammaru. 2012 Degree of specialization is related to body size in herbivorous insects: a phylogenetic confirmation. Evolution 67: 583-589.

Diniz-Filho, J.A.F., M.Á. Rodríguez, L.M. Bini, M.Á. OlallaTarraga, M. Cardillo, J.C. Nabout, J. Hortal and B.A. Hawkins. 2009. Climate history, human impacts and global body size of Carnivora (Mammalia: Eutheria) at multiple evolutionary scales. J. Biogeogr. 36: 2222-2236.

Dynesius, M. and R. Jansson. 2000. Evolutionary consequences of changes in species' geographical distributions driven by Milankovitch climate oscillations. Proc. Nat. Acad. Sci. (USA) 97: 9115-9120.

Fischer, K. and K. Fiedler. 2002. Reaction norms for age and size at maturity in response to temperature: a test of the compound interest hypothesis. Evol. Ecol. 16: 333-349.

Forstmeier W. and H. Schielzeth. 2011. Cryptic multiple hypotheses testing in linear models: overestimated effect sizes and the winner's curse. Behav. Ecol. Sociobiol. 65: 47-55.

Gaston, K.J. and S.L. Chown. 2013. Macroecological patterns in insect body size. In: F.A. Smith and S.K. Lyons (eds.), Animal Body Size: Linking Pattern and Process across Space, Time and Taxonomic Group. University of Chicago Press, Chicago. pp. $13-61$.

Heinrich, B. 1993. The Hot-blooded Insects: Strategies and Mechanisms of Thermoregulation. Harvard University Press, Cambridge.
Hu, J.H., F. Xie, C. Li and J.P. Jiang. 2011. Elevational patterns of species richness, range and body size for spiny frogs. PLoS One 6: e19817.

Jackson, L.S. and P.M. Forster. 2010. An empirical study of geographic and seasonal variations in diurnal temperature range. $J$. Climate 23: 3205-3221.

Janzen, D.H. 1967. Why mountain passes are higher in the tropics. Am. Nat. 101: 233-249.

Kingsolver, J.G., H. A. Woods, L.B. Buckley, K.A. Potter, H.J. MacLean and J.K. Higgins. 2011. Symposium. Complex life cycles and the responses of insects to climate change, $14 \mathrm{pp}$. Integr. Comp. Biol., Oxford Univ. Press. doi: 10.1093/icb/icr015.

Lee, S.Y., G.R. Scott and W.K. Milsom. 2008. Have wing morphology or flight kinematics evolved for extreme high altitude migration in the bar-headed goose? Comp. Biochem. Physiol. (C) 148: 324-331.

Linacre, E. 1982. The effect of altitude on the daily range of temperature. J. Climatol. 2: 375-382.

Lindstroem, J., L. Kaila and P. Niemelä. 1994. Polyphagy and adult body size in geometrid moths. Oecologia 98: 130-132.

Longino, J.T. and R.K. Colwell. 2011. Density compensation, species composition, and richness of ants on a Neotropical elevational gradient. Ecosphere 2: art29.

Luke J., H., J.T. Weir, C. D. Brock, R.E. Glor and W. Challenger 2008. GEIGER: investigating evolutionary radiations. Bioinformatics 24:129-131.

McCain, C.M. and K.B. Knight. 2013. Elevational Rapoport's rule is not pervasive on mountains. Glob. Ecol. Biogeogr. 22: 750-759.

McCain, C.M. 2009. Vertebrate range sizes indicate that mountains may be 'higher' in the tropics. Ecol. Lett. 12: 550-560.

Meiri, S. 2010. Bergmann's rule - what's in a name? Glob. Ecol. Biogeogr. 20: 203-207.

Merckx, T. and E.M. Slade. 2014. Macro-moth families differ in their attraction to light: implications for light-trap monitoring programmes. Ins. Cons. Divers. 7: 453-461.

Olalla-Tárraga, M.A. and M.A. Rodriguez. 2007. Energy and interspecific body size patterns of amphibian faunas in Europe and North America: anurans follow Bergmann's rule, urodeles its converse. Glob. Ecol. Biogeogr. 16: 606-617.

Pagel, M. 1997. Inferring evolutionary processes from phylogenies. Zool. Scr. 26: 331-348.

Paradis, E., J. Claude and K. Strimmer. 2004. APE: analyses of phylogenetics and evolution in R language. Bioinformatics 20: 289-290.

Regier, J.C., C. Mitter, A. Zwick, A.L. Bazinet, M.P. Cummings, A.Y. Kawahara, J.-C. Sohn, D.J. Zwickl, S. Cho, D.R. Davis, J. Baixeras, J. Brown, C. Parr, S. Weller, D.C. Lees and K.T. Mitter. 2013. A large-scale, higher-level, molecular phylogenetic study of the insect order Lepidoptera (moths and butterflies). PLoS One 8: e58568.

Rodríguez, M.Á., M.Á. Olalla-Tárraga and B.A. Hawkins. 2008. Bergmann's rule and the geography of mammal body size in the Western Hemisphere. Glob. Ecol. Biogeogr. 17: 274-283.

Ruggiero, A. and B.A. Hawkins. 2006. Mapping macroecology. Glob. Ecol. Biogeogr. 15: 433-437.

Sandel, B., L. Arge, B. Dalsgaard, R.G. Davies, K.J. Gaston, W.J. Sutherland and J.-C. Svenning. 2011. The influence of late quaternary climate-change velocity on species endemism. Science 334: 660-664.

Sanders, N.J. 2002. Elevational gradients in ant species richness: area, geometry, and Rapoport's rule. Ecography 25: 25-32. 
Stevens, G.C. 1989. The latitudinal gradient in geographical range: how so many species coexist in the tropics. Am. Nat. 133: 240256.

Stevens, G.C. 1992. The elevational gradient in altitudinal range: an extension of Rapoport's latitudinal rule to altitude. Am. Nat. 140: 893-911.

Sullivan, J.B. and W.E. Miller. 2007. Intraspecific body size variation in Macrolepidoptera as related to altitude of capture site and seasonal generation. J. Lepidopt. Soc. 61: 72-77.

Tomašových, A., D. Jablonski, S.K. Berke, A.Z. Krug and J.W. Valentine. 2015. Nonlinear thermal gradients shape broad-scale patterns in geographic range size and can reverse Rapoport's rule. Glob. Ecol. Biogeogr. 24: 157-167.

Truxa, C. and K. Fiedler. 2012. Attraction to light - from how far do moths (Lepidoptera) return to weak artificial sources of light? Europ. J. Entomol. 109: 77-84.

Watt, C., S. Mitchell and V. Salewski. 2010. Bergmann's rule: a concept cluster? Oikos 119: 89-100.

Wiens, J.J., D.D. Ackerly, A.P. Allen, B.L. Anacker, L.B. Buckley, H.V. Cornell, E.I. Damschen, T.J. Davies, J.-A. Grytnes, S.P. Harrison, B.A. Hawkins, R.D. Holt, C.M. McCain and P.R. Stephens. 2010. Niche conservatism as an emerging principle in ecology and conservation biology. Ecol. Lett. 13: 1310-1324.

Woods, H.A. 2013. Ontogenetic changes in the body temperature of an insect herbivore. Funct. Ecol. 27: 1322-1331.

Zamora-Camacho, F.J., S. Reguera and G. Moreno-Rueda. 2014. Bergmann's rule rules body size in an ectotherm: heat conserva- tion in a lizard along a 2200-metre elevational gradient. J. Evol. Biol. 27: 2820-2828.

Zuo, W., M.E. Moses, G.B. West, C. Hou and J.H. Brown. 2012. A general model for effects of temperature on ectotherm ontogenetic growth and development. Proc. Roy. Soc. (B) 279: 18401846.

Received March 4, 2016

Revised May 31, 2016

Accepted June 20, 2016

\section{Appendix: Electronic Supplementary material}

ES1: Sources of trait data, nomenclature and identifications

ES2: Methods of phylogenetic reconstruction

ES3: Phylogenetic tree

ES4: Full results on model selection

ES5: Robustness analyses

ES6: Results based on Worldclim-temperatures

ES7: Local and global pattern of temperature variability as a function of elevation

ES8: Raw data of traits and occurrence for all species (csvformat)

The files may be downloaded from www.akademiai.com. 